\title{
A GENERALIZED PICARD GROUP FOR PRIME RINGS
}

\author{
S. MONTGOMERY \\ University of Southern California \\ Los Angeles, California, U.S.A.
}

Let $R$ be a prime ring. The generalized Picard group is defined to be $p(R)=X$-inn $R / \operatorname{Inn} R$, the quotient group of $X$-inner automorphisms of $R$ modulo the subgroup of inner automorphisms. $X$-inner automorphisms were introduced in ring theory in 1975 [Kh75] and about the same time in $C^{*}$-algebras. They have had many uses in studying fixed rings and noncommutative Galois theory, ideals in crossed products, and in computing automorphism groups of some specific rings. Thus there are a number of papers in the literature describing $X$-inner automorphisms.

In this paper we look more specifically at the quotient group $p(R)$. First we discuss why it is an analog of the classical Picard group (or more precisely the central Picard group). We then give a number of examples of $p(R)$, which can be extracted from the known results on $X$-inner automorphisms. We then return to $p(R)$ and examine how it behaves on the $\operatorname{ring} M_{n}(R)$ of $n \times n$ matrices over $R$. Finally, we include a discussion of the symmetric ring of quotients and the relationship between $X$-inner automorphisms and the "partly inner" automorphisms used in $C^{*}$-algebras.

The author would like to thank R. M. Guralnick for some helpful suggestions in $\$ 3$, particularly concerning the Proposition and Corollary. She also acknowledges support from NSF Grant DMS 87-00641.

\section{$\S 1 . X$-inner automorphisms and invertible ideals}

We first recall the definition of $X$-inner automorphisms. Let $Q_{l}(R)$ denote the left Martindale quotient ring of $R$; that is, $Q_{l}(R)=\underset{\substack{0 \neq I \\ 0 \neq l}}{\operatorname{Hom}}\left({ }_{R} I, R\right)$, the direct

This paper is in final form and no version of it will be submitted for publication elsewhere. 
limit over the nonzero ideals $I$ of $R$, where $\operatorname{Hom}\left({ }_{R} I, R\right)$ denotes the left $R$-module maps from $I$ to $R$. The ring $R$ imbeds into $Q_{l}(R)$ via $r \rightarrow r_{R}$, right multiplication by $r$ on $I=R$. If $\sigma \in$ Aut $R$, then $\sigma$ has a unique extension to $Q_{l}(R)$. We say $\sigma$ is $X$-inner if $\sigma$ becomes inner on $Q_{l}(R)$.

In fact the definition of $X$-inner is left-right symmetric (since $\sigma$ is $X$-inner if and only if there exist $0 \neq a, b, c, d \in R$ such that $a r b=c r^{\sigma} d$, for all $r \in R$ [MP $84]$ ), and thus one could have used the right Martindale quotient ring $Q_{r}(R)$, or the symmetric quotient ring $Q_{s}(R)=Q_{1}(R) \cap Q_{r}(R)$, which is what we shall use here. We will write $Q_{s}(R)=Q(R)$, or just $Q$ when there is no ambiguity about which ring is involved. The center $C$ of $Q$ is called the extended center of $R$, and is known to be a field.

One reference for $Q_{l}(R)$ and $X$-inner automorphisms is [M80]. $Q_{s}(R)$ will be discussed further in Section 4.

We require a few more facts. If $\sigma$ is $X$-inner on $R$, induced by $q \in Q$, then $q R=R q$, that is, $q$ is $R$-normalizing. Conversely, if $0 \neq x \in Q$ is an $R$-normalizing element, then $x$ is a unit in $Q$ and so determines a unique $X$-inner automorphism $\sigma_{x}$, via $x \sigma_{x}(r)=r x$, for all $r \in R$ [M80, p. 43].

We now consider ideals. An $R$-ideal of $Q$ is an $R$-R-subbimodule $I$ of $Q ; I$ is invertible if there exists an $R$-ideal $J$ of $Q$ such that $I J=J I=R$. The set $\mathscr{I}(R)$ of all invertible $R$-ideals of $Q$ is a group under multiplication. An $R$-ideal $I$ is principal if there exists $x \in Q$ such that $I=x R=R x$; if $x \neq 0$, then by the above remark $x^{-1} \in Q$ and so $I$ is invertible. Let $\mathscr{P}(R)$ denote the subgroup of $\mathscr{I}(R)$ of all nonzero principal ideals. $\mathscr{C}(R)$ denotes the subgroup of central principal ideals; that is, $I \in \mathscr{C}(R)$ if $I=c R$, some $c \in C$.

Our first lemma is essentially due to K. A. Brown. Although he states it in the situation when $R$ is Noetherian and $Q$ is the classical quotient ring, the same proof works [B, Lemma 4.2].

LEMMA 1. For any prime ring $R, p(R) \cong \mathscr{P}(R) / \mathscr{C}(R)$.

Proof. Define $\varphi: \mathscr{P}(R) \rightarrow X$-inn $R / \operatorname{Inn} R=p(R)$ by $\varphi(R x)=\sigma_{x} \operatorname{Inn} R$, where $R x=x R$ and $\sigma_{x}$ denotes conjugation by $x$ as above. $\varphi$ is well defined, for if $R x=R y$, then $x=u y$ for some unit $u$ of $R$, and so $\sigma_{x} \equiv \sigma_{y}(\bmod \operatorname{Inn} R)$. Clearly $\varphi$ is onto. Also $\operatorname{Ker} \varphi=\mathscr{C}(R)$, for

$$
\begin{aligned}
I=R x \in \operatorname{Ker} \varphi & \Leftrightarrow \sigma_{x} \in \operatorname{Inn} R \\
& \Leftrightarrow x=c u, \text { for some } c \in C \text { and some unit } u \text { of } R \\
& \Leftrightarrow I=R x=R c \in \mathscr{C}(R) .
\end{aligned}
$$

The lemma motivates our calling $p(R)$ a generalized Picard group, although it is actually closer to the central Picard group [R, p. 320], or central class group [B]. The $p$ also suggests that we are considering principal ideals. As is true classically, a ring $R$ will be considered good if $p(R)$ is "small"; that is, $p(R)$ is finite or at least abelian. 


\section{§ 2. Examples}

As mentioned in the introduction, many examples of $p(R)$ can be obtained from known results on $X$-inner automorphisms. We survey many of those results here.

\section{A. PI rings and Azumaya algebras}

If $R$ is prime PI with center $Z$, and $g \in$ Aut $R$ fixes $Z$, then $g$ fixes the center of $Q=R Z^{-1}$ and so is inner on $Q$ by the Skolem-Noether theorem. That is, $g$ is $X$-inner. Conversely, any $g \in X$-inn $R$ fixes $Z$. Thus $X$-inn $R=A \mathrm{Aut}_{z} R$, and so $p(R)=$ Aut $_{l} R / \operatorname{Inn} R=$ Out $_{z} R$.

If $R$ is in addition an Azumaya algebra of rank $n^{2}$ over $Z$, then Out $_{Z} R$ is abelian and $n$-torsion, by work of Rosenberg-Zelinsky and Knus-Ojanguren (see [KO]). If also $R$ is affine over a field $k$ of characteristic 0 , it is known that $\mathrm{Out}_{Z} R$ is finite.

For general PI rings, however, Out ${ }_{z} R$ is not necessarily well behaved, and can even be a simple group. A subgroup of $\operatorname{Out}_{z} R=p(R)$ is better behaved: let Loc Inn $R$ denote the "locally inner" automorphisms of $R$ : that is, all $\sigma \in \mathrm{Aut}_{z} R$ such that $\sigma$ becomes inner on every localization of $R$ at a maximal ideal of $Z$. Then Loc Inn $R / \operatorname{Inn} R$ is always abelian [C].

\section{B. Free algebras and free products}

If $R$ is a free algebra of rank $\geqslant 2$ over a field $k$, then it is known that $Q(R)=R[\mathrm{Kh} 78]$. Consequently $X$-inn $R \subseteq \operatorname{Inn} R=\langle 1\rangle$ and so $p(R)=\langle 1\rangle$.

More generally, free products (or coproducts) have been studied in the papers [MaM], [LiMa], [Ma]. The best result so far is that if $D$ is a division ring and $R_{1}, R_{2}$ are von Neumann finite $D$-rings, then every $X$-inner automorphism of $R=R_{1} \Perp_{D} R_{2}$ is inner, except for a few special cases [Ma] (the only exception when $D$ is a field and the $R_{i}$ are domains occurs if $\left.\left[R_{1}: D\right]=\left[R_{2}: D\right]=2[\mathrm{MaM}]\right)$. Thus $p(R)=\langle 1\rangle$ for such rings.

We do not know whether or not $Q(R)=R$ in this situation (excluding the special cases).

\section{Enveloping algebras of Lie algebras and Ore extensions}

Let $L$ be a Lie algebra over a field $k$ and $R=U(L)$ its universal enveloping algebra. An element $q \in Q(R)$ is called a semi-invariant for the action of ad $L$ if there exists $\lambda \in L^{*}$, the dual of $L$, such that ad $x(q)=[x, q]=\lambda(x) q$, for all $x \in L$. If $q$ is a semi-invariant, then $q$ is $R$-normalizing, so determines an $X$-inner automorphism $\sigma$ given by $\sigma(x)=q^{-1} x q=x+\lambda(x)$, for all $x \in L$. Conversely, it 
is shown in [M81] that any $X$-inner automorphism is of this form. Since $U(L)$ has no nontrivial units, Inn $R=\langle 1\rangle$, and thus $p(R)=X$-inn $R$ can be identified with an additive subgroup of $L^{*}$. Therefore $p(R)$ is abelian; if also $k$ has characteristic $0, p(R)$ is torsion-free.

For any domain $R$, let $S=R[x ; d]$ be an Ore extension for $d$ a derivation of $R$. In [M83], it is proved that $p(S)$ is an extension of a subquotient of $C-\{0\}$ by a subgroup of $p(R)$. In particular, $p(S)$ is abelian provided $p(R)=1$. A result is also proved for iterated Ore extensions, giving another proof that $p(R)$ is abelian for $R=U(L)$, when $L$ is a solvable Lie algebra.

\section{Group algebras and crossed products}

If $R=k G$ is a prime group algebra over the field $k$, let $W$ denote the set of $X$-inners of $k G$ which normalize the group $k^{*} G$ of trivial units. Then [MP82] it can be proved that $W / W \cap \operatorname{Inn} R$ is a periodic abelian group. If $G$ is a right ordered group, then $W=X$-inn $R$ and thus $p(R)$ is periodic abelian. If also $\Delta(G)=1$, where $\Delta$ is the f.c. subgroup of $G$, then $p(R)=1$. In particular, this applies to the case when $G$ is a free group, although the fact that $p(R)=1$ for $R=k G, G$ free, can also be obtained from the free product results [MaM].

The description of $W$ is also of interest. [MP81] proves that $\sigma \in W \Leftrightarrow \sigma=\sigma_{1} \sigma_{2} \sigma_{3}$, where $\sigma_{1} \in \operatorname{Inn} G, \sigma_{3}$ fixes a subgroup $H$ of $G$ of finite index, and $\sigma_{2}$ is $X$-inner of "scalar type", that is, there exists a linear character $\lambda: G \rightarrow k$ such that $\sigma_{2}(g)=\lambda(g) g$, for all $g \in G$. Such an automorphism is $X$-inner if and only if there exists $\alpha \in k G$ such that $\alpha^{-1} g \alpha=\lambda(g) g$, or $g \alpha g^{-1}=\lambda(g) \alpha$, for all $g$; this says that $\alpha$ is a semi-invariant for the action of Ad $G$, and is analogous to what happened for $U(L)$.

In a related result, [B] considers group rings $R G$ for $R$ a commutative Noetherian UFD and $G$ a dihedral-free polycyclic-by-finite group with $\Delta^{+}(G)=1$. He proves that for such group rings,

$$
p(R G) \cong H^{1}\left(G / C_{G}(\Delta), R^{*} \times \Delta\right)
$$

where $R^{*}$ denotes the units of $R$. As a consequence, he obtains a different proof of a result in [MP83].

$X$-inners of crossed products $R * G$ are studied in [MP86]. When $R$ and $R * G$ are prime, results are obtained about the group $X$ of $X$-inners of $R * G$ which normalize both $R$ and the group of trivial units of $R * G$; again an appropriate quotient of $\mathscr{X}$ is a torsion abelian group. A major special case occurs when $R=U(L)$, an enveloping algebra. Here the skew group ring $H=U(L) G$ is actually a Hopf algebra, provided $G$ acts as automorphisms of $L$. As for enveloping algebras and group algebras, the semi-invariants for the action of ad $H$ on itself give examples of $X$-inner automorphisms of $H$. 


\section{E. Generic matrices and their trace rings}

Let $R=k\left\{X_{1}, \ldots, X_{d}\right\}$ be the ring of $d$ generic $n \times n$ matrices, where $d, n \geqslant 2$. It was proved independently in [LvKh] and [M81] that $X$-inn $R$ is trivial; thus $p(R)=\langle 1\rangle$.

More generally, if $T$ is the trace ring of $R$, then again $X$-inn $T$ is trivial and so $p(T)=\langle 1\rangle$, with one exception: the case $n=d=2$. In this case $X$-inn $T \cong Z_{2} ;$ the unique nontrivial automorphism is given by $X_{i}^{\sigma}=-X_{i}+\left(\operatorname{tr} X_{i}\right) I, i=1,2$. This result is due to [L] in characteristic 0 and to $[\mathrm{GM}]$ in characteristic $p \neq 0$.

F. Finally, $p(R)$ can be arbitrary. In [MP83] it is shown that for any group $G$, there exists a subring $R$ of some group algebra such that $p(R) \cong G$.

\section{$\S$ 3. $p(R)$, module isomorphisms, and matrices}

In this section we return to our general consideration of $p(R)$ and its properties. We first show that $\mathscr{P}(R)$ can be viewed as ideals module-isomorphic to $R$.

Lemma 2. Assume $a \in Q$ such that $I=a R$ is an invertible $R$-ideal of $Q$ and such that $a$ is right regular on $R$. Then $a R=R a$, and thus $a^{-1} \in Q$ and $I \in \mathscr{P}(R)$.

Proof. Since $a \in Q$, there exists an ideal $K$ of $R$ such that $0 \neq a K \subseteq R$. Moreover, since $I$ is an $R$-ideal, $R(a K)=(R a) K \subseteq(a R) K=a K$, and thus $a K$ is an ideal of $R$. Define $f: a K \rightarrow R$ by $f(a k)=k ; f$ is well defined since $a$ is right regular. Clearly $f$ is a right $R$-map, and thus determines an element $\tilde{f} \in Q_{r}(R)$. Letting $a_{L}$ denote left multiplication by $a$, both $f \circ a_{L}$ and $a_{L} \circ f$ are the identity on $a K$. Thus in $Q_{r}, \tilde{f}=\left(\tilde{a}_{L}\right)^{-1}$. But $a \rightarrow a_{L}$ is just the imbedding of $R$ in $Q_{r}$. Thus $a^{-1} \in Q_{r}$.

Now $R a \subseteq a R$ implies $a^{-1} R \subseteq R a^{-1}$, and thus $J=R a^{-1}$ is an $R$-ideal of $Q_{r}$ with $J I=R$. But $I$ is invertible in $Q$, and so in $Q_{r}$; it follows that $J=I^{-1}$. Thus $J \subseteq Q=Q_{s}$ and in particular $a^{-1} \in Q$. Finally, $R=I J=a R a^{-1}$, and so $a R=R a$.

The lemma raises an interesting question: namely, what happens if $I$ is not assumed to be invertible? That is, if $R$ is prime and $a \in Q$ such that $R a \subseteq a R$, when must $a$ be $R$-normalizing? R. Guralnick has proved that $a$ is normalizing if $R$ is a finite module over its center. We do not know what happens when $R$ is an affine PI algebra.

Proposition. For any $I \in \mathscr{I}(R)$, the following are equivalent:

(1) $I \cong R$ as right $R$-modules.

(2) $I \cong R$ as left $R$-modules.

(3) $I \in \mathscr{P}(R)$. 
Proof. Clearly (3) implies (1) and (2), since if $I=R a=a R$ for some unit $a \in Q, I \cong R$ both as a right and left $R$-module.

$(1) \Rightarrow(3)$. Let $\varphi: R \rightarrow I$ be a right $R$-module isomorphism and let $a=\varphi(1) \in I \subseteq Q$. Since $\varphi$ is one-to-one, $a$ is right regular, and since $\varphi$ is onto, $I=a R$. Thus by Lemma $2, a^{-1} \in Q$ and $a R=R a$, proving (3). (2) $\Rightarrow(3)$ uses the left version of Lemma 2.

We now proceed to matrix rings. For convenience we will write $S=M_{n}(R)$, the ring of $n \times n$ matrices over $R$. The proof of the next lemma is straightforward.

LEMMA 3. Let $S=M_{n}(R)$, for $R$ a prime ring.

(1) $Q(S)=M_{n}(Q(R))$.

(2) $I$ is an $S$-ideal of $Q(S) \Leftrightarrow I=M_{n}(J)$, for $J$ an $R$-ideal of $Q$.

(3) If $I=M_{n}(J)$, then $I \in \mathscr{I}(S) \Leftrightarrow J \in \mathscr{I}(R)$.

For any $R$-module $V, V^{(n)}$ denotes the direct sum of $n$ copies of $V$.

COROllary. For any $I \in \mathscr{I}(R)$, the following are equivalent:

(1) $I^{(n)} \cong R^{(n)}$ as right $R$-modules.

(2) $I^{(n)} \cong R^{(n)}$ as left $R$-modules.

(3) $M_{n}(I) \in \mathscr{P}\left(M_{n}(R)\right)$.

Proof. From the Morita correspondence, $I^{(n)} \cong R^{(n)}$ as right $R$-modules if and only if $M_{n}(I) \cong M_{n}(R)$ as right $M_{n}(R)$-modules; similarly on the left. The corollary now follows from the Proposition.

We may now define $\mathscr{I}^{n}(R)$ to be the set of all $I \in \mathscr{I}(R)$ which satisfy any of the equivalent conditions of the corollary. It is easy to see that $\mathscr{I}^{n}(R)$ is a subgroup of $\mathscr{I}(R)$. Note that $\mathscr{I}^{1}(R)=\mathscr{P}(R)$.

THEOREM. $\quad p\left(M_{n}(R)\right) \cong \mathscr{I}^{n}(R) / \mathscr{C}(R)$.

Proof. By Lemma $1, p\left(M_{n}(R)\right) \cong \mathscr{P}\left(M_{n}(R)\right) / \mathscr{C}\left(M_{n}(R)\right)$. Since the center of $M_{n}(Q)$ is just scalar matrices over $C=C(R), M_{n}(I) \in \mathscr{C}\left(M_{n}(R)\right)$ if and only if $I \in \mathscr{C}(R)$. The theorem now follows from the corollary and the definition of $\mathscr{I}^{n}(R)$.

We note an easy consequence of the theorem: $p\left(M_{n}(R)\right)$ will be abelian whenever $\mathscr{I}^{n}(R)$ is abelian. More generally, we are interested in the relationship between $p(R)$ and $p\left(M_{n}(R)\right)$. Since $p(R) \cong \mathscr{I}^{1}(R) / \mathscr{C}(R)$, it is clearly a subgroup of $p\left(M_{n}(R)\right)$. This could of course be seen directly; $X$-inners of $R$ extend to $X$-inners of $S=M_{n}(R)$ by conjugating by the appropriate "scalar" matrix. Identifying $X$-inn $R$ with its image in $X$-inn $S, X$-inn $R \cap \operatorname{Inn} S=\operatorname{Inn} R$. Thus $p(R)=X$-inn $R / \operatorname{Inn} R \subset X$-inn $S / \operatorname{Inn} S=p(S)$. One might hope that in general $p(R)$ would be a normal subgroup of $p\left(M_{n}(R)\right)$. However, this is false.

ExAmple. Let $R$ have Z-basis $\left\{1, x, y, x y \mid x^{2}=-1, y^{2}=-5, x y\right.$ 
$=-x y\} ;$ thus $R$ is an order in a generalized quaternion (division) algebra $D$ and $R$ has center $\mathbf{Z}$. Then $\operatorname{Out}_{\mathbf{z}} R$ is not normal in $\operatorname{Out}_{\mathbf{z}} M_{2}(R)$.

Proof (sketch). There are several ways to see this. First is a direct computation, which was done jointly with D. S. Passman. We show first that Aut $_{z} R=\left\langle\sigma_{1+x}, \sigma_{y}\right\rangle$, where $\sigma_{1+x}$ denotes conjugation by $1+x$ and $\sigma_{y}$ by $y$. Thus Aut $_{\mathbf{z}} R \cong \mathbf{D}_{4}$, the dihedral group of 8 elements. Also Inn $R=\left\langle\sigma_{x}\right\rangle$ and $\sigma_{x}=\left(\sigma_{1}+{ }_{x}\right)^{2}$; consequently Out $_{\mathbf{Z}} R \cong \mathbf{Z}_{2} \times \mathbf{Z}_{2}$.

Letting $S=M_{2}(R)$, extend Aut $_{2} R$ to Aut $S$. We then show that the element

$$
A=\left(\begin{array}{cc}
2 & -1+y \\
1+y & -2
\end{array}\right)
$$

is $S$-normalizing, so $\sigma_{A} \in \operatorname{Aut}_{\mathbf{Z}} S$, and that $\sigma_{A} \sigma_{1+x} \sigma_{A^{-1}}=\sigma_{B}$, where

$$
B=\left(\begin{array}{cc}
1+x(-4+y) & -2 x y \\
2 x y & 1+x(-4-y)
\end{array}\right) \text {. }
$$

So far the arguments are relatively straightforward. However, to finish the proof, a much more tedious computation is needed to show that $\sigma_{B} \notin$ Aut $R \cdot \operatorname{Inn} S$, and so in $\mathrm{Out}_{\mathbf{Z}} S$, conjugation by the image of $\sigma_{A}$ does not normalize $\mathrm{Out}_{\mathbf{z}} S$.

An alternative to the last computation has been suggested by D. Estes and R. Guralnick. Since $Z$ is a PID and $S$ is a $Z$-order, it follows from known results on orders that for any $n$,

$$
\text { Out }_{\mathbf{z}} M_{n}(R) \subset \prod_{p \in \mathbf{Z}} \text { Out } R_{(p)},
$$

where $p$ denotes a prime in $Z$ and $R_{(p)}$ the localization at $p$. One can check that Out $R_{(p)}=\langle 1\rangle$ if $p \neq 2,5$, that Out $R_{(2)} \cong Z_{2}$ and that Out $R_{(5)} \cong S_{3}$. Thus Out $_{\mathbf{z}} S \subseteq \mathrm{Z}_{2} \times S_{3}$. In fact $\mathrm{Out}_{\mathrm{z}} S=\mathrm{Z}_{2} \times S_{3}$, since Out $\mathrm{z} \cong \mathbf{Z}_{2} \times \mathrm{Z}_{2} \subset \mathrm{Out}_{\mathbf{z}} S$ and $\sigma_{A} \notin$ Out $_{\mathbf{z}} R$. Clearly $\mathbf{Z}_{2} \times \mathbf{Z}_{2}$ is not normal in $\mathbf{Z}_{2} \times S_{3}$.

\section{§. Quotient rings, inner automorphisms, and $C^{*}$-algebras}

In this section we discuss in more detail the symmetric ring of quotients and the relationship of $X$-inner automorphisms to the analogous definition for $C^{*}$-algebras.

We first review the multiplier algebra $M(A)$ of an algebra $A[\mathrm{H}] . M(A)$ is defined to be the set of pairs $(f, g)$, where $f$ (respectively $g$ ) is a left (right) $A$-module map of $A$ to itself, satisfying $f(a) b=a g(b)$, for all $a, b \in A . M(A)$ is an algebra under composition of maps. Notice that $A$ imbeds in $M(A)$ via $a \rightarrow\left(a_{R}, a_{L}\right)$, where $a_{R}$ (resp. $\left.a_{L}\right)$ denotes right (left) multiplication by $a$.

Although the ring $Q_{s}(R)$ was defined as $Q_{l}(R) \cap Q_{r}(R)$ in [Kh77], an equivalent formulation with the flavor of multipliers can be given [P]: let $I$, $J$ be nonzero ideals of $R$, and say $f:{ }_{R} I \rightarrow{ }_{R} R$ and $g: J_{R} \rightarrow R_{R}$. The pair $(f, g)$ is 
called balanced if $f(a) b=a g(b)$, for all $a \in I, b \in J$. Then $Q_{s}(R)$ may be defined as the direct limit of the balanced pairs $(f, g)$ over pairs $I, J$ of ideals of $R$ (in fact, one may assume $I=J$ since $I \cap J \neq 0)$. R imbeds in $Q_{s}(R)$ as above, via $r \rightarrow\left(r_{R}, r_{L}\right)$.

It is this point of view which is close to that used in $C^{*}$-algebras. If $A$ is a prime $C^{*}$-algebra, consider the set $\mathscr{S}$ of nonzero closed ideals of $A$. Then $M^{\infty}(A)$ is defined to be the direct limit over $I \in \mathscr{P}$ of the multiplier algebras $M(I)$; this definition is due to G. Pedersen (see [E]). At first glance $M^{\infty}(A)$ appears to differ from $Q_{s}(A)$ in two major ways: first, only closed ideals are used, and second, the maps $f, g$ are $I$-maps from $I$ to $I$ rather than $A$-maps from $A$ to $I$. However, the second difference can be eliminated. For, since any closed ideal $I \neq 0$ is itself a $C^{*}$-algebra, $I^{2}=I$. It is then easy to see that the two kinds of maps are the same. It follows that the only difference between $M^{\infty}(A)$ and $Q_{s}(A)$ is that only closed ideals are used in $M^{\infty}(A)$; in fact, $M^{\infty}(A)$ imbeds into $Q_{s}(A)$.

We may now define the $C^{*}$-analog of $X$-inner automorphisms. A (continuous) automorphism $\sigma$ of a (prime) $C^{*}$-algebra $A$ is called partly inner if $\sigma$ becomes inner when extended to $M^{\infty}(A)$. Such automorphism are used in [Ri] to study prime crossed products, obtaining analogs of the algebraic results.

\section{References}

[B] K. A. B rown, Class groups and automorphism groups of group rings, Glasgow Math. J. 28 (1986), 79-86.

[C] D. 'Г-M. Chi, Automorphisms of prime Azumaya algebras, Comm. Algebra 12 (1984), 12071212 (see also Ph.D. Thesis. Lniversity of Southern California. 1984).

[E] G. A. Elliott, Automorphisms determined hy mulipliers on ideals of a $C^{*}$-algebra, J. Funct. Anal. 23 (1976), 1-10.

[GM] R. M. Guralnick and S. Montgomery, Normalizing elements of the trace ring of generic matrices, Comm. Algebra 17 (1989). 18051813.

[H] G. Hochschild, Cohomology and representations of associative algebras, Duke Math. J. 14 (1947), 921-948.

[KO] M.-A. Knus and M. Ojanguren. Théorie de la Descente et Algèbres d'Azumaya,

[Kh75] V. K. Kharchenko, Generalized identities with automorphisms, Algebra i Logika 14 (1975), $215-237$ (in Russian).

[Kh77] -, Galois theory of semiprime rings, ibid. 16 (1977), 313.363 (in Russian).

[Kh78] -, Algehras of invariants of free algebras, ibid. 17 (1978), 478-487 (in Russian). Lecture Notes in Math. 389, Springer, Berlin 1974.

[L] L. Le B r y n, Trace rings of generic matrices are unique factorization domains, Glasgow Math. J. 28 (1986), 11-13.

[LiMa] A. Lichtman and W. S. Martindale, The normal closure of the coproduct of domains over a division ring, Comm. Algebra 13 (1985), 1643-1664.

[LvKh] I. V. L'vov and V. K. K harchenko, Normal elements of the algebra of generic matrices are central, Sibirsk. Mat. Zh. 23 (1) (1982), 193195 (in Russian). 
[Ma] W. S. Martindale, The normal closure of the coproduct of rings over a division ring, Trans. Amer. Math. Soc. 293 (1986), 303-317.

[MaM] W. S. Martindale and S. Montgomery, The normal closure of coproducts of domains, J. Algebra 82 (1983), 1-17.

[M80] S. Montgomery, Fixed Rings of Finite Automorphism Groups of Associative Rings, Lecture Notes in Math. 818, Springer, Berlin 1980.

[M81] -, X-inner automorphisms of filtered algebras, Proc. Amer. Math. Soc. 83 (1981), 263-268.

[M83] -, X-inner automorphisms of filtered algebras $I I$, ibid. 87 (1983), 569-575.

[MP81] S. Montgomery and D. S. Passman, $X$-inner automorphisms of group rings, Houston J. Math. 7 (1981), 395-402.

[MP82] -, -, X-inner automorphisms of group rings $I I$, ibid. 8 (1982), 537-544.

[MP84] -, -, Outer Galois theory of prime rings, Rocky Mountain J. Math. 14 (1984), $305-318$.

[MP86] - , , X-inner automorphisms of crossed products and semi-invariants of Hopf algebras, Israel J. Math. 55 (1986), 33-57.

[P] D. S. Passman, Computing the symmetric ring of quotients, J. Algebra 105 (1987), 207--235.

[Pe] G. Pedersen, $C^{*}$-Algebras and their Automorphism Groups, Academic Press, New York 1979.

[R] I. Reiner, Maximal Orders, Academic Press, New York 1975.

[Ri] M. A. Rieffel, Actions of finite groups on $C^{*}$-algebras, Math. Scand. 47 (1980), 157 176. 